\title{
Black Hole Results from XMM-Newton
}

\author{
Norbert Schartel ${ }^{1}$ \\ ${ }^{1} X M M-N e w t o n$ Science Operations Centre, ESA, Villafranca del Castillo, Apartado 78, E-28691 Villanueva de la Cañada \\ Madrid, Spain
}

Corresponding author: Norbert.Schartel@sciops.esa.int

\begin{abstract}
XMM-Newton is one of the most successful science missions of the European Space Agency. Since 2003 every year about 300 articles are published in refereed journals making directly use of XMM-Newton data. All XMM-Newton calls for observing proposals are highly oversubscribed by factors of six and more. In the following some scientific highlights of XMM-Newton observations of black holes are summarized.
\end{abstract}

Keywords: X-ray - AGN - black hole.

\section{Introduction}

XMM-Newton ([1]) is the second cornerstone of European Space Agency's (ESA) Horizon 2000 Science Programme, providing an observatory-class X-ray facility. The spacecraft was launched by an Ariane 5 on 10 December 1999. The observatory provides simultaneous non-dispersive spectroscopic imaging and timing (European Photon Imaging Camera; EPIC, [2] and [3]), medium resolution dispersive spectroscopy (Reflection Grating Spectrometer; RGS, [4]) and optical/UV imaging, spectroscopy and timing from a co-aligned telescope (Optical Monitor; OM, [5]). The three X-ray mirrors ([6]) in combination with the cameras of EPIC offer a large effective area over the energy range from $300 \mathrm{eV}$ to $12 \mathrm{keV}$, up to $2500 \mathrm{~cm}^{2}$ at $1.5 \mathrm{keV}$ and $\sim 1800 \mathrm{~cm}^{2}$ at $5 \mathrm{keV}$. The scientific potential of the effective area may be illustrated by the first observation of an evolving dust-scattered X-ray halo around a gamma ray burst ([7]). Each of the two modules of the RGS cover the energy range from $\sim 0.4 \mathrm{keV}$ to $2.2 \mathrm{keV}$ with an effective area of $60 \mathrm{~cm}^{2}$ at $15 \AA$.

\section{Scientific Highlights}

Scientific highlights resulting from the first decade of XMM-Newton and Chandra observations can be found in [8]. In the following I list a number of highlights from XMM-Newton observations of black holes. In this paper I have focused on some of the most exciting discoveries in this field, which also received wide publicity in public relations announcements by ESA.

\subsection{Galactic black holes and ultraluminous X-ray sources}

Globular clusters (GC), containing thousands of stars packed within tens of light years, were considered as a possible breeding ground for black holes. A rival hypothesis suggests that black holes are ejected through close star encounters and consequently GCs are devoid of black holes. XMM-Newton observations of NGC 4472 allowed the first detection of a black hole in a GC ([9]) excluding the latter hypothesis. Ultraluminous X-ray sources (ULX) were proposed to harbour intermediate-mass black holes, which provides the link between stellar mass black holes and supermassive black holes (SMBH) in the centres of galaxies. XMM-Newton and Chandra observations of CXOM31 J004253.1+411422 in Andromeda allowed to connect this ULX to Low Mass X-ray Binaries ([10], implying accretion onto a stellar-mass black hole in the Eddington regime. A second ULX found in Andromeda, XMMU J004243.6+412519, allowed to observe the emission of the accretion disk in X-rays together with the emission form its jets in radio ([11]). The experimental key for both observations was the low absorbing column density towards Andromeda whereas absorption is a major obstacle of ULX observations in our own Galaxy. [12] found an intermediate-mass black hole in NGC 1313. The X-ray spectra of the ULX can be described with a power law plus an accretion disk $(k T \cong$ $150 \mathrm{eV}$ ) implying a mass of $\cong 10^{3} M_{\odot}$. A intermediatemass black hole with $m>500 \mathrm{M}_{\odot}$ could be associated with an ULX in ESO 243-49 based on luminosity variations observed by XMM-Newton ([13]). 
Black Hole Results from XMM-Newton

\section{$2.2 \quad$ The strong gravitational field}

Currently, X-ray observations are the only way to observe the strong gravitational field in the direct vicinity of black holes and neutron stars ([14]). Special and general relativistic effects distort the spectra of particles orbiting black holes depending on the orbital parameters and the black hole's spin. Theoretical discussion can be found in [15], [16], [17] [18] or more recently in [19] and [20]. Experimentally the iron $\mathrm{K} \alpha$ line is most studied as there are no lines of abundant elements nearby. Early examples are the XMM-Newton observations of the Galactic black hole XTE J1650-500 in outburst ([21]) and the Active Galactic Nuclei (AGN) MGC-6-30-15 in low state ([22]). Both spectra are explained by a fast spinning black hole and the extraction and dissipation of rotational energy from it. The simultaneous XMM-Newton and NuSTAR observation of NGC 1365 revealed that reflection from an ionized disk readily explains the spectra taken by both satellites ([23]). The XMM-Newton spectra of the Galactic black hole, GX 339-4 in outburst ([24]) is an example of a black holes with almost maximum spin. The observation of $1 \mathrm{H} 0706-495$ is unique as it shows not only the iron $\mathrm{K} \alpha$, but also the iron $\mathrm{L} \alpha$ line ([25]). In addition the light cure shows the expected characteristic variability of reflection from an ionized disk (compare also [26], [27], [28], [29]).

\subsection{Active Galactic Nuclei (AGN) in low states}

Following [30] X-ray spectra of AGNs are composed of a power-law continuum emitted above a black hole plus reflection from an ionized disk. During the low state the continuum emission region moves nearer to the black hole and gravitational bending affects its light path. Observationally, during low states the continuum emission appears suppressed whereas the reflected emission appears constant or even enhanced, compare also [31]. The most intensive studied AGN in low state with XMM-Newton is PG 2112+059, where an additional layer of ionized material was used to favour the reflection interpretation versus alternative scenarios ([32], [33] and [34]). [35] used variability considerations to discriminate the reflection interpretation versus an absorption scenario for PG $0844+349$ in an X-ray weak state. And [36] could demonstrate for the low state observation of 1H0707-495 reflected emission within one gravitational radius of the event horizon of the black hole.

\subsection{Aspects of variability near Supermassive Black Holes (SMBHs)}

Whereas quasi-periodic-oscillations (QPO) are well established in X-ray binaries for almost 30 years, QPOs remained elusive in AGNs. XMM-Newton measured a $\sim 1$ hour QPO for RE J1034+396 ([37]). [38] found evidence for orbital motion of material close to the central black hole of Mrk 766. A XMM-Newton observation allowed [39] to observe a co-rotating flare at a distance of only 3.5 to 8 Schwarzschild radii to the SMBH of NGC 3516.

\subsection{Energy budget, winds and outflows}

[40] established for the fist time simultaneous spectral energy distributions for the majority of the [41] reverberation mapped sample of AGN based on XMMNewton EPIC and OM measurements. [42] used XMMNewton observations to show that radio-galaxies produce sufficient mechanical energy to unbind a significant fraction of the intra-group medium, an effect which is negligible in massive clusters of galaxies. Combining high resolution RGS spectra with sensitive light-curves of EPIC, [43] demonstrated an accretion-disk origin for the two warm absorber winds in NGC 4051. 1H 0707495 shows a mildly relativistic, highly ionized outflow which changed its velocity from about $0.11 \mathrm{c}$ to $0.17 \mathrm{c}$ between 2008 January and 2010 September ([44]). Ultrafast outflows are present in $>35 \%$ of radio-quiet AGN observed with XMM-Newton, providing a significant contribution to the AGN cosmological feedback ([45], [46], [47]).

\subsection{Flares and tidal disruption events}

[48] observed several peaks in the power density spectrum of the X-ray light curve of the SMBH in the Galactic Centre during which period a bright X-ray flare was detected ([49]). Theoretical studies revealed a previously unknown topological structure inherent to black holes with high spin: in a small region near the event horizon of the spinning black hole the orbital velocity decreases for decreasing orbital radius ([50]). This effect is now rightfully known as the Aschenbach effect ([51]). [52] could identify a tidal disruption event based on ROSAT, Chandra and XMM-Newton observations. Suzaku and XMM-Newton observations taken shortly after the occurrence of the tidal disruption event Swift J164449.3+573451 reveal a 200-second X-ray quasiperiodicity ([53]). This QPO might be explained with the forming of an accretion disc or precession of the jet. 


\subsection{Deep fields and cosmology}

A total of 1000 AGN detections from a variety of ROSAT, XMM-Newton and Chandra surveys allowed [54] to obtain for the first time reliable space densities for low-luminosity (Seyfert-type) X-ray sources at high cosmological redshifts. Their evolutionary behaviour shows strong dependency on the X-ray luminosity and differs from the dependency found for high luminosity AGNs and quasars. XMM-Newton allows the detection of quasars at highest redshift, e.g. SDSS J104433012502 at $\mathrm{z}=5.80([55])$. The spacecraft could even establish an X-ray spectrum of SDSS J1030+0524 at $\mathrm{z}=6.30$ ([56]). An ionized iron $\mathrm{K} \alpha$ absorption edge in the X-ray spectrum of APM $08279+5255$ allowed to obtain an, at the time of publication, highly interesting constrain on the age of the universe ([57]).

\section{Discussion and Conclusions}

Since 2003 every year about 300 articles are published in refereed journals making directly use of XMM-Newton data. All XMM-Newton calls for observing proposals are highly oversubscribed by factors of six and more. Within ESA's mission extension scheme all missions are evaluated every 2 years and possibly extended by 4 years subjected to midterm confirmation. XMMNewton is funded up to end of 2016 subject to midterm confirmation and further extension discussion in 2014. Currently, the XMM-Newton mission is implementing four-reaction-wheel operation schemata, which will reduce fuel consumption significantly. The envisaged operation mode will allow technically operating the mission up to 2026 .

\section{Acknowledgement}

I thank the organizers of the Workshop and especially Franco Giovannelli for giving him the opportunity to show highlights of XMM-Newton black hole observations. And I thank the anonymous referee for many fruitful comments and suggestions.

\section{References}

[1] F. Jansen et al. XMM-Newton observatory. I. The spacecraft and operations. $A_{-} A, 365:$ L1-L6, January 2001.

[2] M. J. L. Turner et al. The European Photon Imaging Camera on XMM-Newton: The MOS cameras: The MOS cameras. A\&\&A, 365:L27-L35, January 2001. doi:10.1051/0004-6361:20000087
[3] L. Strüder et al. The European Photon Imaging Camera on XMM-Newton: The pn-CCD camera. $A \mathscr{E} A$, 365:L18-L26, January 2001.

[4] J. W. den Herder et al. The Reflection Grating Spectrometer on board XMMNewton. $\quad A \& A$, 365:L7-L17, January 2001. doi:10.1051/0004-6361:20000058

[5] K. O. Mason et al. The XMM-Newton optical/UV monitor telescope. A\&A , 365:L36-L44, January 2001. doi:10.1051/0004-6361:20000044

[6] B. Aschenbach. In-orbit performance of the XMMNewton x-ray telescopes: images and spectra. In P. Gorenstein and R. B. Hoover, editors, Society of Photo-Optical Instrumentation Engineers (SPIE) Conference Series, volume 4496 of Society of Photo-Optical Instrumentation Engineers (SPIE) Conference Series, pages 8-22, January 2002. doi:10.1038/nature08690

[7] S. Vaughan et al. The Discovery of an Evolving Dust-scattered X-Ray Halo around GRB 031203. ApJ, 603:L5-L8, March 2004. doi:10.1038/nature05434

[8] M. Santos-Lleo et al. The first decade of science with Chandra and XMM-Newton. Nature, 462:997-1004, December 2009.

[9] T. J. Maccarone et al. A black hole in a globular cluster. Nature, 445:183-185, January 2007.

[10] A. Kaur et al. CXOM31 J004253.1+411422: the first ultraluminous X-ray transient in M 31. $A \mathscr{G} A$ , 538:A49, February 2012.

[11] M. J. Middleton et al. Bright radio emission from an ultraluminous stellar-mass microquasar in M 31. Nature, 493:187-190, January 2013. doi:10.1038/nature11697

[12] J. M. Miller et al. X-Ray Spectroscopic Evidence for Intermediate-Mass Black Holes: Cool Accretion Disks in Two Ultraluminous X-Ray Sources. ApJ, 585:L37-L40, March 2003.

[13] S. A. Farrell et al. An intermediate-mass black hole of over 500 solar masses in the galaxy ESO243-49. Nature, 460:73-75, July 2009. doi:10.1038/nature08083

[14] O. K. Madej and P. G. Jonker. Discovery of a broad O VIII Ly $\alpha$ line in the ultracompact X-ray binary 4U 1543-624. MNRAS, 412:L11-L15, March 2011.

[15] A. C. Fabian et al. X-ray fluorescence from the inner disc in Cygnus X-1. MNRAS, 238:729-736, May 1989. 
Black Hole Results from XMM-Newton

[16] A. Laor, H. Netzer, and T. Piran. Massive thin accretion discs. II - Polarization. MNRAS, 242:560569, February 1990.

[17] K. Beckwith and C. Done. Extreme gravitational lensing near rotating black holes. MNRAS, 359:1217-1228, June 2005.

[18] M. Dovčiak, V. Karas, and T. Yaqoob. An Extended Scheme for Fitting X-Ray Data with Accretion Disk Spectra in the Strong Gravity Regime. ApJS, 153:205-221, July 2004.

[19] T. Dauser et al. Broad emission lines for a negatively spinning black hole. MNRAS, 409:15341540, December 2010.

[20] T. Dauser et al. Irradiation of an accretion disc by a jet: general properties and implications for spin measurements of black holes. MNRAS, 430:16941708, April 2013.

[21] J. M. Miller et al. Evidence of Spin and Energy Extraction in a Galactic Black Hole Candidate: The XMM-Newton/EPIC-pn Spectrum of XTE J1650500. ApJ, 570:L69-L73, May 2002.

[22] J. Wilms et al. XMM-EPIC observation of MCG-630-15: direct evidence for the extraction of energy from a spinning black hole? MNRAS, 328:L27L31, December 2001.

[23] G. Risaliti et al. A rapidly spinning supermassive black hole at the centre of NGC1365. Nature, 494:449-451, February 2013. doi:10.1038/nature11938

[24] J. M. Miller et al. Evidence of Black Hole Spin in GX 339-4: XMM-Newton/EPIC-pn and RXTE Spectroscopy of the Very High State. ApJ, 606:L131-L134, May 2004.

[25] A. C. Fabian et al. Broad line emission from iron Kand L-shell transitions in the active galaxy 1H0707495. Nature, 459:540-542, May 2009.

[26] A. Zoghbi et al. Broad iron L line and X-ray reverberation in 1H0707-495. MNRAS, 401:2419-2432, February 2010.

[27] A. Zoghbi, P. Uttley, and A. C. Fabian. Understanding reverberation lags in 1H0707-495. MNRAS, 412:59-64, March 2011.

[28] L. Miller et al. X-ray reverberation in 1H0707495 revisited. MNRAS, 408:1928-1935, November 2010.
[29] E. Kara et al. The closest look at 1H0707-495: X-ray reverberation lags with $1.3 \mathrm{Ms}$ of data. $M N$ RAS, 428:2795-2804, February 2013.

[30] G. Miniutti and A. C. Fabian. A light bending model for the X-ray temporal and spectral properties of accreting black holes. MNRAS, 349:14351448, April 2004.

[31] L. C. Gallo. Connection between spectral complexity and X-ray weakness: testing the reflection interpretation. Astronomische Nachrichten, 327:1076, December 2006. doi:10.1002/asna. 200610695

[32] N. Schartel et al. The XMM-Newton view of three $\mathrm{X}$-ray weak quasars: Iron emission and strong ionized absorption. A\&A , 433:455-465, April 2005.

[33] N. Schartel et al. XMM-Newton observation of the deep minimum state of PG 2112+059. A spectrum dominated by reflection from the accretion disk? A\&SA , 474:431-441, November 2007.

[34] N. Schartel et al. A long hard look at the minimum state of PG 2112+059 with XMM-Newton. A\&BA , 512:A75, March 2010. doi:10.1002/asna. 200610695

[35] L. C. Gallo et al. The quasar PG 0844+349 in an X-ray weak state. $M N R A S, 412: 161-170$, March 2011.

[36] A. C. Fabian et al. 1H 0707-495 in 2011: an X-ray source within a gravitational radius of the event horizon. MNRAS, 419:116-123, January 2012.

[37] M. Gierliński et al. A periodicity of 1hour in X-ray emission from the active galaxy $\mathrm{RE}$ J1034+396. Nature, 455:369-371, September 2008. doi:10.1038/nature07277

[38] T. J. Turner et al. Evidence for orbital motion of material close to the central black hole of Mrk 766 . $A \mathscr{E} A$, 445:59-67, January 2006.

[39] K. Iwasawa, G. Miniutti, and A. C. Fabian. Flux and energy modulation of redshifted iron emission in NGC 3516: implications for the black hole mass. MNRAS, 355:1073-1079, December 2004.

[40] R. V. Vasudevan and A. C. Fabian. Simultaneous X-ray/optical/UV snapshots of active galactic nuclei from XMM-Newton: spectral energy distributions for the reverberation mapped sample. $M N$ RAS, 392:1124-1140, January 2009. 
[41] B. M. Peterson et al. Central Masses and BroadLine Region Sizes of Active Galactic Nuclei. II. A Homogeneous Analysis of a Large ReverberationMapping Database. ApJ, 613:682-699, October 2004.

[42] S. Giodini et al. Radio Galaxy Feedback in Xray-selected Groups from COSMOS: The Effect on the Intracluster Medium. ApJ, 714:218-228, May 2010.

[43] Y. Krongold et al. The Compact, Conical, Accretion-Disk Warm Absorber of the Seyfert 1 Galaxy NGC 4051 and Its Implications for IGMGalaxy Feedback Processes. ApJ, 659:1022-1039, April 2007.

[44] T. Dauser et al. Spectral analysis of 1H 0707-495 with XMM-Newton. MNRAS, 422:1914-1921, May 2012.

[45] F. Tombesi et al. Evidence for ultra-fast outflows in radio-quiet AGNs. I. Detection and statistical incidence of $\mathrm{Fe} \mathrm{K}$-shell absorption lines. $A \mathscr{E} A$, 521:A57, October 2010.

[46] F. Tombesi et al. Evidence for Ultra-fast Outflows in Radio-quiet Active Galactic Nuclei. II. Detailed Photoionization Modeling of Fe K-shell Absorption Lines. ApJ, 742:44, November 2011.

[47] F. Tombesi et al. Evidence for ultrafast outflows in radio-quiet AGNs - III. Location and energetics. MNRAS, 422:L1, May 2012.

[48] B. Aschenbach et al. X-ray flares reveal mass and angular momentum of the Galactic Center black hole. AEA , 417:71-78, April 2004.

[49] D. Porquet et al. XMM-Newton observation of the brightest X-ray flare detected so far from Sgr A*. $A \mathscr{E} A$, 407:L17-L20, August 2003.
[50] B. Aschenbach. Measuring mass and angular momentum of black holes with high-frequency quasiperiodic oscillations. A\&A , 425:1075-1082, October 2004 .

[51] Z. Stuchlík et al. Aschenbach effect: Unexpected topology changes in the motion of particles and fluids orbiting rapidly rotating Kerr black holes. Phys. Rev. D, 71(2):024037, January 2005. doi:10.1103/PhysRevD.71.024037

[52] S. Komossa et al. A Huge Drop in the X-Ray Luminosity of the Nonactive Galaxy RX J1242.61119A, and the First Postflare Spectrum: Testing the Tidal Disruption Scenario. ApJ, 603:L17-L20, March 2004.

[53] R. C. Reis et al. A 200-Second Quasi-Periodicity After the Tidal Disruption of a Star by a Dormant Black Hole. Science, 337:949-, August 2012. doi:10.1126/science. 1223940

[54] G. Hasinger, T. Miyaji, and M. Schmidt. Luminosity-dependent evolution of soft X-ray selected AGN. New Chandra and XMM-Newton surveys. $A \mathscr{E} A$, 441:417-434, October 2005.

[55] W. N. Brandt et al. An XMM-Newton Detection of the $\mathrm{Z}=5.80 \mathrm{X}$-Ray-Weak Quasar SDSSP J104433.04-012502.2. AJ, 121:591-597, February 2001.

[56] D. Farrah et al. The X-Ray Spectrum of the $\mathrm{z}=6.30$ QSO SDSS J1030+0524. ApJ, 611:L13-L16, August 2004 .

[57] G. Hasinger, N. Schartel, and S. Komossa. Discovery of an Ionized Fe K Edge in the $\mathrm{z}=3.91$ Broad Absorption Line Quasar APM 08279+5255 with XMM-Newton. ApJ, 573:L77-L80, July 2002. 